ANNALES

POLONICI MATHEMATICI

$92.1(2007)$

\title{
A symmetry problem
}

\author{
by A. G. RAmm (Manhattan, KS)
}

Abstract. Consider the Newtonian potential of a homogeneous bounded body $D \subset \mathbb{R}^{3}$ with known constant density and connected complement. If this potential equals $c /|x|$ in a neighborhood of infinity, where $c>0$ is a constant, then the body is a ball. This known result is now proved by a different simple method. The method can be applied to other problems.

1. Introduction. Consider a bounded domain $D \subset \mathbb{R}^{3}$ with a connected complement and $C^{1, \lambda}$-smooth boundary $S$. The smoothness assumptions on $S$ can be weakened, but this is not the point of this paper. Let $B_{R}$ be a ball of radius $R$, containing $D$, and $B_{R}^{\prime}$ be its complement in $\mathbb{R}^{3}$. We denote by $S^{2}$ a unit sphere, and by $\ell$ a unit vector. Let $N$ be the outer unit normal to $S$. Denote by $\chi$ the characteristic function of $D$, and by $\mathcal{N}$ the set of harmonic functions in $B_{R}$. Let the center $O$ of $B_{R}$ be the origin, and suppose it lies at the center of mass of $D$.

Consider the Newtonian potential

$$
u(x):=\int_{D} \frac{d y}{|x-y|},
$$

where we have assumed that the density of the mass distribution in $D$ is 1 .

Assume that

$$
u(x)=c|x|^{-1} \quad \text { in } B_{R}^{\prime},
$$

where $c=$ const. Then the question is:

Does this imply that $D$ is a ball?

It is well known and easy to prove that if $D$ is a ball $B_{a}$ of radius $a$, then $u(x)=c|x|^{-1}$ in $B_{a}^{\prime}$, and $c=\left|B_{a}\right|$, where $\left|B_{a}\right|$ is the volume of this ball, $\left|B_{a}\right|=4 \pi a^{3} / 3$, so $a=(3 c / 4 \pi)^{1 / 3}$. In [1] and [5] one can find different proofs of the fact that the answer to the above question is yes. An especially

2000 Mathematics Subject Classification: 31A25, 35J05, 35J15.

Key words and phrases: Newtonian potential. 
simple proof, due to D. Zagier, is given at the end of this paper (see also review [5], where one can find this proof).

Our goal is to give a simple new proof of this result by a method which can be used in other problems (see, e.g., [4]). The literature on potential theory and inverse potential theory is quite large, and we only mention a few references [1]-[3], [5], where the reader can find additional bibliography, and [4], where an argument similar to the one we use was applied to the study of the Pompeiu problem.

We do not attempt to make the weakest assumption about the smoothness of the boundary of $D$. In Zagier's proof, given at the end of this paper, no smoothness of the boundary is assumed.

We prove the following theorem:

THEOREM 1. Under the above assumptions, if $u(x)=c|x|^{-1}$ in $B_{R}^{\prime}$, then $D$ is a ball of radius $a=(3 c / 4 \pi)^{1 / 3}$.

This result is proved in Section 2.

\section{Proofs}

Proof of Theorem 1. We have

$$
\Delta u=-4 \pi \chi \quad \text { in } \mathbb{R}^{3} .
$$

Multiply (2) by a harmonic function $h \in \mathcal{N}$ and integrate over $B_{R}$ to get

$$
-4 \pi \int_{D} h(x) d x=\int_{B_{R}} h \Delta u d x=\int_{B_{R}} u \Delta h d x+I,
$$

where we have used Green's formula, and set

$$
I:=\int_{\partial B_{R}}\left(h u_{r}-u h_{r}\right) d s, \quad u_{r}:=\frac{\partial u}{\partial r}=\left.\frac{\partial u}{\partial N}\right|_{\partial B_{R}},
$$

where $\partial B_{R}$ is the boundary of $B_{R}$. By our assumption,

$$
u_{r}=-c R^{-2}, \quad u=c R^{-1} \quad \text { on } \partial B_{R} .
$$

We also have

$$
\int_{\partial B_{R}} h_{r} d s=0, \quad \frac{1}{4 \pi R^{2}} \int_{\partial B_{R}} h d s=h(0),
$$

where we have used the mean value theorem for harmonic functions and the formula

$$
\int_{\partial B_{R}} h_{r} d s=\int_{B_{R}} \Delta h d x=0,
$$

which is valid for harmonic functions $h$. Therefore, $I=c_{1} h(0)$, where $c_{1}$ is 
a constant, and (3) implies

$$
\int_{D} h(x) d x=c_{2} h(0) \quad \forall h \in \mathcal{N}, \quad c_{2}:=-\frac{c_{1}}{4 \pi} .
$$

If $h \in \mathcal{N}$, then $h(g x) \in \mathcal{N}$ for any rotation $g$. Let us check that if $g=g(\phi)$ is the rotation through the angle $\phi$ about the straight line passing through the origin in the direction $\ell$, then

$$
\left.\frac{d(g(\phi) x)}{d \phi}\right|_{\phi=0}=[\ell, x]
$$

where $[\ell, x]$ is the cross product. To check (5), choose the coordinate system with $z$-axis along $\ell$, and write the matrix $g$ of the $\phi$-rotation about the $z$-axis:

$$
g:=g(\phi)=\left(\begin{array}{ccc}
\cos \phi & -\sin \phi & 0 \\
\sin \phi & \cos \phi & 0 \\
0 & 0 & 1
\end{array}\right)
$$

Then

$$
\left.\frac{d g}{d \phi}\right|_{\phi=0}=\left(\begin{array}{ccc}
0 & -1 & 0 \\
1 & 0 & 0 \\
0 & 0 & 0
\end{array}\right):=G .
$$

Thus, $G x=[\ell, x]$ in the chosen coordinate system. This formula does not depend on the choice of coordinate system, so (5) is verified.

A proof of (5), similar to the one used in mechanics for the proof of the conservation of angular momentum, is also possible.

Replacing $h(x)$ by $h(g(\phi) x)$ in $(4)$, differentiating with respect to $\phi$, then setting $\phi=0$, and using (5), one gets

$$
\int_{D} \nabla h \cdot[\ell, x] d x=0
$$

where $\cdot$ is the dot product of two vectors. Note that

$$
\nabla h(x) \cdot[\ell, x]=\nabla \cdot h(x)[\ell, x] .
$$

Therefore, using the divergence theorem, one rewrites (6) as

$$
\int_{S} h(s) N_{s} \cdot[\ell, s] d s=\ell \cdot \int_{S} h(s)\left[s, N_{s}\right] d s=0, \quad \forall h \in \mathcal{N}, \forall \ell \in S^{2},
$$

where $N_{s}$ is the outer unit normal to $S$ at the point $s$. Since $\ell$ is arbitrary, equation (7) implies

$$
\int_{S} h(s)\left[s, N_{s}\right] d s=0, \quad \forall h \in \mathcal{N} .
$$


We claim that the set of restrictions of all harmonic functions $h \in \mathcal{N}$ to $S$ is dense in $L^{2}(S)$. For the convenience of the reader, this claim is verified after the proof of Theorem 1 is finished. Thus, (8) implies

$$
\left[s, N_{s}\right]=0 \quad \forall s \in S .
$$

Let us prove that (9) implies that $S$ is a sphere. Let $\mathbf{r}=\mathbf{r}(p, q)$ be a parametric equation of $S$. Then $s=\mathbf{r}(p, q)$ and $N_{s}=\left[\mathbf{r}_{p}, \mathbf{r}_{q}\right] /\left|\left[\mathbf{r}_{p}, \mathbf{r}_{q}\right]\right|$. Thus, (9) implies

$$
0=\left[\mathbf{r},\left[\mathbf{r}_{p}, \mathbf{r}_{q}\right]\right]=\mathbf{r}_{p} \mathbf{r} \cdot \mathbf{r}_{q}-\mathbf{r}_{q} \mathbf{r} \cdot \mathbf{r}_{p} .
$$

Since the surface $S$ is assumed $C^{1, \lambda}$-smooth, the normal $N_{s}$ is well defined at every point $s \in S$, and the vectors $\mathbf{r}_{p}$ and $\mathbf{r}_{q}$ are linearly independent. Thus, (10) implies

$$
\mathbf{r} \cdot \mathbf{r}_{q}=\mathbf{r} \cdot \mathbf{r}_{p}=0
$$

It follows from (11) that

$$
\mathbf{r} \cdot \mathbf{r}=a^{2},
$$

where $a>0$ is a constant. This is an equation of a sphere of radius $a$, centered at the origin, i.e., at the center $O$ of mass of $D$. So, $D$ is a ball of radius $a$ centered at $O$. In our argument we do not assume that $D$ is connected.

Theorem 1 is proved.

Let us now verify the claim that the set of restrictions of all harmonic functions $h \in \mathcal{N}$ to $S$ is dense in $L^{2}(S)$. Assuming the contrary, one concludes that there exists an $f \in L^{2}(S)$ such that

$$
\int_{S} f(s) h(s) d s=0 \quad \forall h \in \mathcal{N} .
$$

Take

$$
h(s)=\int_{S_{m}} \frac{\mu(x) d x}{|x-s|},
$$

where $m>R$, and $\mu \in L^{2}\left(S_{m}\right)$ is arbitrary. Then $h$ is harmonic in $B_{R}$, and since $\mu$ is arbitrary, equation (13) implies

$$
v(x):=\int_{S} f(s)|x-s|^{-1} d s=0 \quad \forall x \in S_{m} .
$$

The function $v$ is a single-layer potential which vanishes on $S_{m}$. Thus, it vanishes everywhere outside $S_{m}$, and consequently, everywhere outside $S$. Since $v$ is continuous in $\mathbb{R}^{3}$, and vanishes everywhere outside $S$, it vanishes on $S$. A function $v$ which is harmonic in $D$ and vanishes on $S$ must vanish in $D$. So, $v=0$ in $D$ and in $D^{\prime}:=\mathbb{R}^{3} \backslash D$. By the jump relation for the normal derivative of $v$ across the boundary $S$, one gets

$$
0=\left(v_{N}\right)^{+}-\left(v_{N}\right)^{-}=4 \pi f,
$$


where $\left(v_{N}\right)^{ \pm}$is the limiting value of the normal derivative $v_{N}$ from the inside (resp. outside) of $D$. So, $f=0$, which proves the claim.

REMARK. Equation (12) has been derived under the assumption that the origin of the coordinate system is fixed: in this coordinate system $u(x)=$ $c /|x|$ in $B_{R}^{\prime}$. Therefore the surfaces satisfying (12) in which $a=$ const $>0$, can be concentric spheres. There cannot be more than two neighboring concentric spheres with different mass densities, because the density of the mass distribution in $D$ is assumed constant. There cannot be two such spheres, i.e., $D$ cannot be a spherical shell, because in this case the domain $D^{\prime}$ is not connected, contrary to our assumption. Thus, $S$ can only be one sphere, and $D$ can only be a ball.

D. Zagier's proof ([5]). Assume that $D$ is connected. If $u(x)=$ $\int_{D} d y /|x-y|=c /|x|$ in $B_{R}^{\prime}$, then $u(x)=c /|x|$ in $D^{\prime}$ by the unique continuation theorem for harmonic functions. Taking gradient, one gets

$$
\int_{D} \frac{d y(x-y)}{|x-y|^{3}}=\frac{c x}{|x|^{3}}
$$

in $D^{\prime}$. Taking the dot product with $x$, one gets

$$
\int_{D} \frac{d y\left(x^{2}-y \cdot x\right)}{|x-y|^{3}}=\frac{c}{|x|}=\int_{D} \frac{d y}{|x-y|}=\int_{D} \frac{d y|x-y|^{2}}{|x-y|^{3}}, \quad x \in D^{\prime} .
$$

This implies

$$
\int_{D} \frac{d y\left(-y^{2}+y \cdot x\right)}{|x-y|^{3}}=0 \quad \text { in } D^{\prime}
$$

Let $B \subset D$ be the largest ball, inscribed in $D$ and centered at the origin, and $B^{c}:=D \backslash B$. One has

$$
\int_{B} \frac{d y\left(-y^{2}+y \cdot x\right)}{|x-y|^{3}}=0 \text { in } B^{\prime} .
$$

Subtract this from the similar formula for $D$ to get

$$
\int_{B^{c}} \frac{d y\left(-y^{2}+y \cdot x\right)}{|x-y|^{3}}=0 \quad \text { in } D^{\prime} .
$$

Since $-y^{2}+y \cdot x \leq 0$ if $y \in B^{c}, x \in D^{\prime}$, one concludes that $\left|B^{c}\right|=0$, so $D=B$. Here $\left|B^{c}\right|$ is the Lebesgue measure (volume) of the set $B^{c}$.

\section{References}

[1] D. Aharonov, M. Schiffer and L. Zalcman, Potato kugel, Israel J. Math. 40 (1981), 331-339. 
[2] A. Margulis, Equivalence and uniqueness in the inverse problem of potential theory for homogeneous starlike bodies, Dokl. Akad. Nauk SSSR 312 (1990), 577-580 (in Russian).

[3] A. G. Ramm, Inverse Problems, Springer, New York, 2005.

[4] - Necessary and sufficient condition for a domain, which fails to have Pompeiu property, to be a ball, J. Inverse Ill-Posed Problems 6 (1998), 165-171.

[5] L. Zalcman, Some inverse problems of potential theory, in: Contemp. Math. 63, Amer. Math. Soc., 1987, 337-350.

Mathematics Department

Kansas State University

Manhattan, KS 66506-2602, U.S.A.

E-mail: ramm@math.ksu.edu

Received 6.12.2006

and in final form 20.4.2007 